\title{
TOWARDS THE FULL MORDELL-LANG CONJECTURE FOR DRINFELD MODULES
}

\author{
DRAGOS GHIOCA
}

\begin{abstract}
Let $\phi$ be a Drinfeld module of generic characteristic, and let $X$ be a sufficiently generic affine subvariety of $\mathbb{G}_{a}^{g}$. We show that the intersection of $X$ with a finite rank $\phi$-submodule of $\mathbb{G}_{a}^{g}$ is finite.
\end{abstract}

\section{INTRODUCTION}

In [18, McQuillan proved the Mordell-Lang conjecture in its most general form.

Theorem 1.1 (The full Mordell-Lang theorem). Let $G$ be a semi-abelian variety defined over a number field $K$. Let $X \subset G$ be a $K^{\text {alg }}$-subvariety, and let $\Gamma \subset G\left(K^{\text {alg }}\right)$ be a finite rank group (i.e. $\Gamma$ lies in the divisible hull of a finitely generated subgroup of $\left.G\left(K^{\text {alg }}\right)\right)$. Then there exist algebraic subgroups $B_{1}, \ldots, B_{l}$ of $G$ and there exist $\gamma_{1}, \ldots, \gamma_{l} \in \Gamma$ such that

$$
X\left(K^{\mathrm{alg}}\right) \cap \Gamma=\bigcup_{i=1}^{l}\left(\gamma_{i}+B_{i}\left(K^{\mathrm{alg}}\right)\right) \cap \Gamma .
$$

We note that in Theorem 1.1 if $X$ does not contain any translate of a positive dimensional algebraic subgroup of $G$, then the full Mordell-Lang theorem says that $X\left(K^{\text {alg }}\right) \cap \Gamma$ is finite. Also, a particular case of the full Mordell-Lang theorem (in the case $\Gamma$ is the torsion subgroup $G_{\text {tor }}$ of $G$ ) is the Manin-Mumford theorem, which was first proved by Raynaud [19].

Faltings $[8$ proved the Mordell-Lang conjecture for finitely generated subgroups $\Gamma$ of abelian varieties $G$. His proof was extended by Vojta 23 to finitely generated subgroups of semi-abelian varieties $G$. Finally, McQuillan [18 extended Vojta's result to finite rank subgroups $\Gamma$ of semi-abelian varieties $G$. Later, Rössler [20] provided a simplified proof of McQuillan's extension in which he used uniformities for the intersection of translates of a fixed subvariety $X \subset G$ with the torsion subgroup of the semi-abelian variety $G$. Essentially, Rössler showed that the full Mordell-Lang conjecture follows from the Mordell-Lang statement for finitely generated subgroups, combined with a uniform Manin-Mumford statement as proved by Hrushovski [17.

It is important to note that the exact translation of the Mordell-Lang conjecture to semi-abelian varieties in characteristic $p$ is false due to the presence of isotrivial varieties. However, Hrushovski [16] saved the Mordell-Lang theorem for finitely generated subgroups of semi-abelian varieties in characteristic $p$ by treating isotrivial varieties as special. The isotrivial case was treated by Rahim Moosa and the author in [12] where it was obtained a full Mordell-Lang statement for isorivial semi-abelian varieties in characteristic $p$. On the other hand, if we replace $G$ by a power $\mathbb{G}_{a}^{g}$ of the additive group scheme, then the exact translation of the Mordell-Lang conjecture either fails (in characteristic 0) or it is trivially true (in characteristic $p$ ).

Inspired by the analogy between abelian varieties in characteristic 0 and Drinfeld modules of generic characteristic, Denis [6 proposed that analogs of the Manin-Mumford and Mordell-Lang theorems hold for such Drinfeld modules $\phi$ acting on $\mathbb{G}_{a}^{g}$ (in characteristic $p$ ). Denis conjectures ask for describing the intersection of an affine subvariety $X \subset \mathbb{G}_{a}^{g}$ with a finite rank $\phi$-submodule $\Gamma$ of $\mathbb{G}_{a}^{g}$. Using methods of model theory, combined with some clever number theoretical arguments, Scanlon 21] proved the Denis-ManinMumford conjecture. In 9], the author proved the Denis-Mordell-Lang conjecture for finitely generated $\phi$-modules $\Gamma$ under two mild technical assumptions. In this paper, we extend our result from [9] to finite rank $\phi$-submodules $\Gamma$.

We also note that recently there have been significant progress on establishing additional links between classical diophantine results over number fields and similar statements for Drinfeld modules. The author proved in [10] an equidistribution statement for torsion points of a Drinfeld module, which is similar to the equidistribution statement established by Szpiro-Ullmo-Zhang [22] (which was later extended by Zhang [26] to a full proof of the famous Bogomolov conjecture). Also, Breuer [3] proved a special case of the 
André-Oort conjecture for Drinfeld modules, while special cases of this conjecture in the classical case of a number field were proven by Edixhoven-Yafaev [7] and Yafaev [25]. Bosser [2] proved a lower bound for linear forms in logarithms at an infinite place associated to a Drinfeld module (similar to the classical result obtained by Baker [1] for usual logarithms, or by David [4 for elliptic logarithms). Bosser's result was used by Thomas Tucker and the author in [13] to establish certain equidistribution and integrality statements for Drinfeld modules. Moreover, Bosser's result is quite possibly true also for linear forms in logarithms at finite places for a Drinfeld module. Assuming this last statement, Thomas Tucker and the author proved in [14] the analog of Siegel's theorem for finitely generated $\phi$-submodules. We believe that our present paper provides an additional proof of the fact that the Drinfeld modules represent the right arithmetic analog in characteristic $p$ for semi-abelian varieties in characteristic 0 .

The plan for our paper is as follows: in Section 2 we provide the basic notation for our paper, while in Section 3 we prove our main result (Theorem 3.1).

\section{The Mordell-Lang theorem for Drinfeld modules}

First we note that all subvarieties appearing in this paper are considered to be closed. We define next the notion of a Drinfeld module.

Let $p$ be a prime and let $q$ be a power of $p$. Let $C$ be a projective non-singular curve defined over $\mathbb{F}_{q}$. Let $A$ be the ring of $\mathbb{F}_{q}$-valued functions defined on $C$, regular away from a fixed closed point $\infty \in C$. Let $K$ be a finitely generated field extension of the fraction field $\operatorname{Frac}(A)$ of $A$. We let $K^{\text {alg }}$ be a fixed algebraic closure of $K$, and let $K^{\text {sep }}$ be the separable closure of $K$ inside $K^{\text {alg }}$.

We define the operator $\tau$ as the Frobenius on $\mathbb{F}_{q}$, extended so that for every $x \in K^{\text {alg }}$, we have $\tau(x)=x^{q}$. Then for every subfield $L \subset K^{\text {alg }}$, we let $L\{\tau\}$ be the ring of polynomials in $\tau$ with coefficients from $L$ (the addition is the usual addition, while the multiplication is given by the usual composition of functions).

Following Goss [15, we call a Drinfeld module of generic characteristic defined over $K$ a morphism $\phi: A \rightarrow K\{\tau\}$ for which the coefficient of $\tau^{0}$ in $\phi_{a}$ is $a$ for every $a \in A$, and there exists $a \in A$ such that $\phi_{a} \neq a \tau^{0}$. For the remainder of this paper, unless otherwise stated, $\phi: A \rightarrow K\{\tau\}$ is a Drinfeld module of generic characteristic.

A Drinfeld module $\psi: A \rightarrow K^{\text {alg }}\{\tau\}$ is isomorphic to $\phi$ (over $K^{\text {alg }}$ ) if there exists a nonzero $\gamma \in K^{\text {alg }}$ such that for every $a \in A$, we have $\psi_{a}=\gamma^{-1} \phi_{a} \gamma$.

For every field extension $K \subset L$, the Drinfeld module $\phi$ induces an action on $\mathbb{G}_{a}(L)$ by $a * x:=\phi_{a}(x)$, for each $a \in A$. Let $g$ be a fixed positive integer. We extend diagonally the action of $\phi$ on $\mathbb{G}_{a}^{g}$.

The subgroups of $\mathbb{G}_{a}^{g}\left(K^{\mathrm{alg}}\right)$ invariant under the action of $\phi$ are called $\phi$-submodules. For a $\phi$-submodule $\Gamma$, its full divisible hull is

$$
\Gamma \otimes_{A} \operatorname{Frac}(A):=\left\{x \in \mathbb{G}_{a}^{g}(K) \mid \text { there exists } 0 \neq a \in A \text { such that } \phi_{a}(x) \in \Gamma\right\} .
$$

We define the rank of a $\phi$-submodule $\Gamma \subset \mathbb{G}_{a}^{g}\left(K^{\mathrm{alg}}\right)$ as $\operatorname{dim}_{\operatorname{Frac}(A)} \Gamma \otimes_{A} \operatorname{Frac}(A)$.

Definition 2.1. An algebraic $\phi$-submodule of $\mathbb{G}_{a}^{g}$ is an algebraic subgroup of $\mathbb{G}_{a}^{g}$ invariant under $\phi$.

Denis proposed in Conjecture 2 of [ $[$ ] the following problem.

Conjecture 2.2 (The full Denis-Mordell-Lang conjecture). Let $X \subset \mathbb{G}_{a}^{g}$ be an affine variety defined over $K^{\text {alg. }}$. Let $\Gamma$ be a finite rank $\phi$-submodule of $\mathbb{G}_{a}^{g}\left(K^{\text {alg }}\right)$. Then there exist algebraic $\phi$-submodules $B_{1}, \ldots, B_{l}$ of $\mathbb{G}_{a}^{g}$ and there exist $\gamma_{1}, \ldots, \gamma_{l} \in \Gamma$ such that

$$
X\left(K^{\text {alg }}\right) \cap \Gamma=\bigcup_{i=1}^{l}\left(\gamma_{i}+B_{i}\left(K^{\text {alg }}\right)\right) \cap \Gamma .
$$

Before stating our result, we need to introduce the following notion.

Definition 2.3. We call the modular transcendence degree of $\phi$ the smallest integer $d \geq 1$ such that a Drinfeld module isomorphic to $\phi$ is defined over a field of transcendence degree $d$ over $\mathbb{F}_{q}$.

In [9] (see Theorem 4.11), the author proved the following result towards Conjecture 2.2 . 
Theorem 2.4. With the above notation, assume in addition that the modular transcendence degree of $\phi$ is at least 2. Let $X \subset \mathbb{G}_{a}^{g}$ be an affine subvariety defined over $K^{\text {alg }}$ such that there is no positive dimensional algebraic subgroup of $\mathbb{G}_{a}^{g}$ whose translate lies inside $X$. Let $\Gamma$ be a finitely generated $\phi$-submodule of $\mathbb{G}_{a}^{g}\left(K^{\mathrm{alg}}\right)$. Then $X\left(K^{\text {alg }}\right) \cap \Gamma$ is finite.

In Theorem 3.1 we extend the previous result to all finite rank $\phi$-submodules $\Gamma$.

Remark 2.5. We have two technical conditions in Theorem 2.4 which we will keep also in our extension from Theorem 3.1. The condition that $\phi$ has modular transcendence degree at least equal to 2 is a mild technical condition, however necessary due to the methods employed in [9]. The condition that $X$ does not contain any translate of a positive dimensional algebraic subgroup of $\mathbb{G}_{a}^{g}$ is satisfied by all sufficiently generic affine subvarieties $X$.

\section{Proof of our main Result}

We continue with the notation from Section 2, We define the torsion submodule of $\phi$ as

$$
\phi_{\text {tor }}=\left\{x \in \mathbb{G}_{a}^{g}\left(K^{\text {alg }}\right) \mid \text { there exists } a \in A \backslash\{0\} \text { such that } \phi_{a}(x)=0\right\} .
$$

Next we state our main result.

Theorem 3.1. Let $K$ be a finitely generated field of characteristic $p$ and let $g$ be a positive integer. Let $\phi: A \rightarrow K\{\tau\}$ be a Drinfeld module of generic characteristic. Assume the modular transcendence degree of $\phi$ is at least 2 . Let $X \subset \mathbb{G}_{a}^{g}$ be an affine subvariety defined over $K^{\text {alg }}$ such that there is no positive dimensional algebraic subgroup of $\mathbb{G}_{a}^{g}$ whose translate lies inside $X$. Let $\Gamma$ be a finitely generated $\phi$-submodule of $\mathbb{G}_{a}^{g}(K)$, and let $\Gamma^{\prime}:=\Gamma \otimes_{A} \operatorname{Frac}(A)$. Then $X\left(K^{\text {alg }}\right) \cap \Gamma^{\prime}$ is finite.

In our proof of Theorem 3.1 we need a uniform version of Scanlon's result from [21]. He proved the Manin-Mumford theorem for Drinfeld modules in the following form (see his Theorem 1).

Theorem 3.2. Let $\phi: A \rightarrow K\{\tau\}$ be a Drinfeld module and let $X \subset \mathbb{G}_{a}^{g}$ be an affine variety defined over $K^{\mathrm{alg}}$. Then there exist algebraic $\phi$-submodules $B_{1}, \ldots, B_{l}$ of $\mathbb{G}_{a}^{g}$ and there exist $\gamma_{1}, \ldots, \gamma_{l} \in \phi_{\text {tor }}^{g}$ such that

$$
X\left(K^{\text {alg }}\right) \cap \phi_{\text {tor }}^{g}=\bigcup_{i=1}^{l}\left(\gamma_{i}+B_{i}\left(K^{\text {alg }}\right)\right) \cap \phi_{\text {tor }}^{g} .
$$

In Remark 19 from [21, Scanlon notes that his Manin-Mumford theorem for Drinfeld modules holds uniformly in algebraic families of varieties, i.e. if $X$ varies inside an algebraic family of varieties, then there exists a uniform bound on the degrees of the Zariski closures of $X\left(K^{\text {alg }}\right) \cap \phi_{\text {tor }}^{g}$. In particular, we obtain the following uniform statement for translates of $X$.

Corollary 3.3. With the notation for $\phi$ and $X$ as in Theorem 3.2, assume in addition that $X$ contains no translate of a positive dimensional algebraic subgroup of $\mathbb{G}_{a}^{g}$. Then there exists a positive integer $N$ such that for every $x \in \mathbb{G}_{a}^{g}\left(K^{\mathrm{alg}}\right)$, the set $\left(x+X\left(K^{\mathrm{alg}}\right)\right) \cap \phi_{\mathrm{tor}}^{g}$ has at most $N$ elements.

Proof. Because $X$ contains no translate of a positive dimensional algebraic subgroup of $\mathbb{G}_{a}^{g}$, then for every $x \in \mathbb{G}_{a}^{g}\left(K^{\text {alg }}\right)$, the algebraic $\phi$-modules $B_{i}$ appearing in the intersection $\left(x+X\left(K^{\text {alg }}\right)\right) \cap \phi_{\text {tor }}^{g}$ are all 0 dimensional. In particular, the set $\left(x+X\left(K^{\text {alg }}\right)\right) \cap \phi_{\text {tor }}^{g}$ is finite. Thus, using the uniformity obtained by Scanlon for his Manin-Mumford theorem, we conclude that the cardinality of $\left(x+X\left(K^{\text {alg }}\right)\right) \cap \phi_{\text {tor }}^{g}$ is uniformly bounded by some positive integer $N$, independent of $x$.

We will also use the following fact in the proof of our Theorem 3.1 .

Fact 3.4. Let $\phi: A \rightarrow K\{\tau\}$ be a Drinfeld module. Then for every positive integer d, there exist finitely many torsion points $x$ of $\phi$ such that $[K(x): K] \leq d$.

Proof. If $x \in \phi_{\text {tor }}$, then the canonical height $\widehat{\mathrm{h}}(x)$ of $x$ (as defined in [5] and [24]) equals 0. Also, as shown in [5], the difference between the canonical height and the usual Weil height is uniformly bounded on $K^{\text {alg }}$. Actually, Denis [5] proves this last statement under the hypothesis that $\operatorname{trdeg}_{\mathbb{F}_{q}} K=1$. However, his proof easily generalizes to fields $K$ of arbitrarily finite transcendence degree. For this we need the construction of a coherent good set of valuations on $K$ as done in [1] (see also the similar construction of heights from 
24]). Essentially, a coherent good set $U_{K}$ of valuations on $K$ is a set of defectless valuations satisfying a product formula on $K$ (for more details, we refer the reader to Sections 2 and 3 of [11]). Then Fact 3.4 follows by noting that there are finitely many points of bounded Weil height and bounded degree over the field $K$ (using Northcott's theorem applied to the global function field $K$ ).

Moreover, Corollary 4.22 of [11] provides an effective upper bound on the size of the torsion of $\phi$ over any finite extension $L$ of $K$ in terms of $\phi$ and the number of places of $L$ lying above places in $U_{K}$ of bad reduction for $\phi$. Because for each field $L$ such that $[L: K] \leq d$, and for each place $v \in U_{K}$, there are at most $d$ places $w$ of $L$ lying above $v$, we conclude that there exists an upper bound for the size of torsion of $\phi$ over all field extensions of degree at most $d$ over $K$ in terms of $\phi, d$ and the number of places in $U_{K}$ of bad reduction for $\phi$.

Proof of Theorem 3.1. Because $\phi$ has generic characteristic, $\Gamma^{\prime} \subset \mathbb{G}_{a}^{g}\left(K^{\text {sep }}\right)$. Hence $X\left(K^{\text {alg }}\right) \cap \Gamma^{\prime}=X\left(K^{\text {sep }}\right) \cap$ $\Gamma^{\prime}$.

We note that our theorem is equivalent with showing that if $X$ is a positive dimensional irreducible subvariety of $\mathbb{G}_{a}^{g}$ satisfying the hypothesis of Theorem 3.1, then $X\left(K^{\mathrm{sep}}\right) \cap \Gamma^{\prime}$ is not Zariski dense in $X$. Moreover, at the expense of moding out through the stabilizer $\operatorname{Stab}(X)$ of $X$ (which is a finite group according to the hypothesis of Theorem 3.1), we may assume $\operatorname{Stab}(X)$ is trivial. Note that after moding out through the (finite) group $\operatorname{Stab}(X)$, the variety $X$ is still positive dimensional and irreducible. Finally, at the expense of replacing $K$ by a finite extension, we may assume that $X$ is defined over $K$, and also $\Gamma \subset \mathbb{G}_{a}^{g}(K)$.

Our proof follows the argument from [12] (which in turn was inspired by the argument from [20]). We assume by contradiction that $X\left(K^{\text {alg }}\right) \cap \Gamma^{\prime}$ is Zariski dense in $X$.

We claim that for every $x \in \Gamma^{\prime}$ and for every $\sigma \in \operatorname{Gal}\left(\mathrm{K}^{\mathrm{sep}} / \mathrm{K}\right)$, we have $\sigma(x)-x \in \phi_{\mathrm{tor}}^{g}$. Indeed, because $x \in \Gamma^{\prime}$, then there exists $0 \neq a \in A$ such that $\phi_{a}(x) \in \Gamma$. Because $\Gamma \subset \mathbb{G}_{a}^{g}(K)$ and $\phi$ is defined over $K$, then

$$
\phi_{a}(\sigma(x))=\sigma\left(\phi_{a}(x)\right)=\phi_{a}(x) .
$$

Hence $\phi_{a}(\sigma(x)-x)=0$, as desired. Therefore, if $x \in X\left(K^{\text {sep }}\right)$, then $\sigma(x)-x \in(-x+X) \cap \phi_{\text {tor }}^{g}$ (we also used that $X$ is defined over $K$, and so, $\sigma(x) \in X$ ). Using Corollary 3.3 we obtain an upper bound on the number of conjugates of $x$, which gives us an upper bound, say $N$, for $[K(x): K]$. Implicitly, we also get $[K(\sigma(x)): K] \leq N$. Because

$$
K(\sigma(x)-x) \subset K(x, \sigma(x)),
$$

we conclude $[K(\sigma(x)-x): K] \leq N^{2}$. Using that $\sigma(x)-x \in \phi_{\text {tor }}^{g}$, and using Fact [3.4, we conclude that the set

$$
\left\{\sigma(x)-x \mid x \in \Gamma^{\prime} \text { and } \sigma \in \mathrm{Gal}\left(\mathrm{K}^{\mathrm{sep}} / \mathrm{K}\right)\right\} \text { is finite. }
$$

Assuming that $X\left(K^{\text {sep }}\right) \cap \Gamma^{\prime}$ is Zariski dense in $X$ (and also using that $X$ is irreducible), then either $X(K) \cap \Gamma^{\prime}$ is Zariski dense in $X$, or $\left(X\left(K^{\text {sep }}\right) \backslash X(K)\right) \cap \Gamma^{\prime}$ is Zariski dense in $X$. If the former statement holds, then $X$ has a Zariski dense intersection with a finitely generated group, as $\Gamma^{\prime} \cap \mathbb{G}_{a}^{g}(K)$ is a finite rank subgroup of the tame module $\mathbb{G}_{a}^{g}(K)$ (see Theorem 1 of [24]). By Theorem 2.4, $X(K) \cap\left(\Gamma^{\prime} \cap \mathbb{G}_{a}^{g}(K)\right.$ ) is finite and hence it cannot be dense in $X$ (because $X$ is assumed to be positive dimensional). Therefore the latter case of the above dichotomy should occur. Using (1), we conclude that there exists a nonzero torsion point $y \in \phi_{\text {tor }}^{g}$ such that the set

$$
\left\{x \in X\left(K^{\mathrm{sep}}\right) \cap \Gamma^{\prime} \mid \sigma(x)-x=y \text { for some } \sigma \in \operatorname{Gal}\left(\mathrm{K}^{\mathrm{sep}} / \mathrm{K}\right)\right\}
$$

is Zariski dense in $X$. Therefore, $y \in \operatorname{Stab}(X)$, contradicting the fact that $X$ has trivial stabilizer. This concludes the proof of Theorem 3.1

\section{REFERENCES}

[1] A. Baker, Transcendental number theory. Cambridge University Press, London-New York, 1975. x+147 pp.

[2] V. Bosser, Minorations de formes linéaires de logarithmes pour les modules de Drinfeld. (French) [Lower bounds of linear forms in logarithms for Drinfeld modules] J. Number Theory 75 (1999), no. 2, 279-323.

[3] F. Breuer, The André-Oort conjecture for products of Drinfeld modular curves. J. reine angew. Math. 579 (2005), 115-144.

[4] S. David, Minorations de formes linéaires de logarithmes elliptiques. (French) [Lower bounds for linear forms in elliptic logarithms] Mém. Soc. Math. France (N.S.) 62 (1995), iv+143 pp.

[5] L. Denis, Hauteurs canoniques et modules de Drinfeld. (French) [Canonical heights and Drinfeld modules] Math. Ann. 294 (1992), no. 2, 213-223. 
[6] L. Denis, Diophantine geometry on Drinfeld modules. The arithmetic of function fields (Columbus, OH, 1991), 285-302, Ohio State Univ. Math. Res. Inst. Publ., 2, de Gruyter, Berlin, 1992.

[7] B. Edixhoven and A. Yafaev, Subvarieties of Shimura type. Ann. of Math. (2) 157 (2003), no. 2, $621-645$.

[8] G. Faltings, The general case of S. Lang's conjecture. Barsotti Symposium in Algebraic Geometry (Abano Terme, 1991), 175-182, Perspect. Math., 15, Academic Press, San Diego, CA, 1994.

[9] D. Ghioca, The Mordell-Lang Theorem for Drinfeld modules, Internat. Math. Res. Notices, 53 (2005), $3273-3307$.

[10] D. Ghioca, Equidistribution for torsion points of a Drinfeld module. Math. Ann. 336 (2006), 841-865.

[11] D. Ghioca, The Lehmer inequality and the Mordell-Weil theorem for Drinfeld modules. J. Number Theory 122 (2007), 37-68.

[12] D. Ghioca and R. Moosa, Division points on subvarieties of isotrivial semiabelian varieties. to appear in International Mathematics Research Notices.

[13] D. Ghioca and T. J. Tucker, Equidistribution and integrality for Drinfeld modules. submitted for publication, available online at http://www.math.mcmaster.ca/ dghioca/papers/papers.html.

[14] D. Ghioca and T. J. Tucker, Siegel's Theorem for Drinfeld modules. preprint, available online at http://www.math.mcmaster.ca/ dghioca/papers/papers.html

[15] D. Goss, Basic structures of function field arithmetic, Ergebnisse der Mathematik und ihrer Grenzgebiete (3) [Results in Mathematics and Related Areas (3)], 35. Springer-Verlag, Berlin, 1996.

[16] E. Hrushovski, The Mordell-Lang conjecture for function fields, J. Amer. Math. Soc. 9 (1996), no. 3, 667-690.

[17] E. Hrushovski, The Manin-Mumford conjecture and the model theory of difference fields. Annals of Pure and Applied Logic 112 (2001), 43-115.

[18] M. McQuillan, Division points on semi-abelian varieties. Invent. Math. 120 (1995), no. 1, 143-159.

[19] M. Raynaud, Sous-variétés d'une variété abélienne et points de torsion. (French) [Subvarieties of an abelian variety and torsion points]. Arithmetic and Geometry, Vol. I, 327-352, Prog. Math., 35, Birkhauser, Boston, MA, 1983.

[20] D. Rössler, An afterthought on the generalized Mordell-Lang conjecture. to appear in the Proceedings of the workshop on Model Theory, Algebraic and Analytic Geometry (Newton Institute, Cambridge, 11-15 July 2005).

[21] T. Scanlon, Diophantine geometry of the torsion of a Drinfeld module. J. Number Theory 97 (2002), no. 1, 10-25.

[22] L. Szpiro, E. Ullmo and S. Zhang, Équirépartition des petits points. (French) [Uniform distribution of small points]. Invent. Math. 127 (1997), no. 2, 337-347.

[23] P. Vojta, Integral points on subvarieties of semiabelian varieties. I. Invent. Math. 126 (1996), no. 1, 133-181.

[24] J. T.-Y. Wang, The Mordell-Weil theorems for Drinfeld modules over finitely generated function fields, Manuscripta math. 106 (2001), 305-314.

[25] A. Yafaev, A conjecture of Yves André's. Duke Math. J. 132 (2006), no. 3, 393-407.

[26] S. Zhang, Equidistribution of small points on abelian varieties. Ann. of Math. (2) 147 (1998), no. 1, $159-165$.

Dragos Ghioca, Department of Mathematics \& Statistics, Hamilton Hall, Room 218, McMaster University, 1280 Main Street West, Hamilton, Ontario L8S 4K1, Canada

E-mail address: dghioca@math.mcmaster.ca 